Pacific Journal of Mathematic 


\title{
SPECTRAL ANALYSIS IN SPACES OF VECTOR VALUED FUNCTIONS
}

\author{
YITZHAK WEIT
}

Spectral analysis properties of $L_{1}^{H}(\boldsymbol{R})$, where $H$ is a separable Hilbert space, are investigated. It is proved that spectral analysis holds for $L_{1}^{H}(R)$ if and only if $H$ is finite-dimensional. The one-sided analogue of Wiener's theorem for some subgroups of the Euclidean motion group, is obtained.

1. Introduction. Let $A$ be a Banach space and $F$ a class of bounded linear transformations of $A$ into itself. Following [2] we say that spectral analysis holds for $A$ if every proper closed subspace of $A$, invariant under $F$, is included in a closed maximal invariant subspace of $A$.

The case where $A$ is the Banach space of sequences summable with weights and $F$ is the class of the translation operators was studied in [2].

We are going to study the problem of spectral analysis with $A$ being the Banach space $L_{1}^{I I}(\boldsymbol{R})$ of functions defined on $\boldsymbol{R}$, taking values in a separable Hilbert space $H$, and $F$ is the class of translations by the group $\boldsymbol{R}$.

Wiener's classical theorem states that spectral analysis holds for $L_{1}^{H}(\boldsymbol{R})$ where $H$ is one-dimensional.

Our main goal is to show that spectral analysis holds for $L_{1}^{H}(\boldsymbol{R})$, if and only if, $H$ is finite-dimensional.

In $\S 2$ we characterize the minimal $w^{*}$-closed, translation invariant subspaces of $L_{\infty}^{H}(\boldsymbol{R})$, the dual space of $L_{1}^{H}(\boldsymbol{R})$.

Spectral analysis in the finite-dimensional case is considered in $\S 3$. In $\S 4$ we construct a $w^{*}$-closed invariant subspace of $L_{\infty}^{I I}(\boldsymbol{R})$ which does not contain a nontrivial, minimal, $w^{*}$-closed, invariant subspace. One-sided spectral analysis in subgroups of the motion group, is studied in $\S 5$.

For $x \in H$ let $\|x\|=(x, x)^{1 / 2}$ denote the norm of $x$. For $f \in L_{\infty}(\boldsymbol{R})$, let $\operatorname{Sp}(f)$ denote the spectrum of $f$.

2. Minimal invariant subspaces. The minimal invariant $w^{*}$ closed subspace of $L_{\infty}^{I I}(\boldsymbol{R})$ are characterized as follows:

THEOREM 1. Let $H$ be a separable Hilbert space with the basis $\left\{e_{n}\right\}_{n=1}^{\infty}$. Then the function $f \in L_{\infty}^{H}(\boldsymbol{R}), f \neq 0$ generates a minimal, $w^{*}$-closed, invariant subspace, if and only if 


$$
\left(f(x), e_{n}\right)=a_{n} e^{i \lambda x} \quad(n=1,2, \cdots,)
$$

for some $\lambda \in \boldsymbol{R}$ and $\left\{a_{n}\right\}_{n=1}^{\infty} \in l_{2}$.

Proof. Let $f_{n}(x)=\left(f(x), e_{n}\right)$ for $n=1,2, \cdots$, .

If $f_{n}(x)=a_{n} e^{i \lambda x}$ then, obviously, the invariant subspace generated by $f$ is one-dimensional.

To prove the "only if" part, let $M$ denote the $w^{*}$-closed, invariant subspace generated by $f, f \in L_{\infty}^{I I}(\boldsymbol{R})$. Suppose that $\lambda_{1} \in$ $\operatorname{Sp}\left(f_{k}\right), \lambda_{2} \in \operatorname{Sp}\left(f_{m}\right)$ where $m \neq k$ and $\lambda_{1}<\lambda_{2}$. Let $\phi \in L_{1}(\boldsymbol{R})$ be such that Supp $\hat{\phi}=\left[r_{1}, r_{2}\right]$ where $r_{1}<\lambda_{1}<r_{2}<\lambda_{2}$. Let $g \in L_{\infty}^{H}(\boldsymbol{R})$ be the function $g(x)=\int_{-\infty}^{\infty} f(x-\alpha) \phi(\alpha) d \alpha$. Let $h \in L_{1}(\boldsymbol{R})$ with Supp $\hat{h} \subset\left(r_{2}, \infty\right)$, such that $\int_{-\infty}^{\infty} f_{m}(x) h(x) d x \neq 0$. Then, for $\psi \in L_{1}^{H}(\boldsymbol{R})$, where $\left(\psi(x), e_{m}\right)=$ $h(x)$ and $\left(\psi(x), e_{n}\right)=0$ for $n \neq m$, we have

$$
\int_{-\infty}^{\infty}(g(x-\alpha), \psi(x)) d x=\int_{-\infty}^{\infty} g_{m}(x-\alpha) h(x) d x=0
$$

for all $\alpha \in \boldsymbol{R}$, where $g_{m}(x)=\left(g_{m}(x), e_{m}\right)$. On the other hand, we have $\int_{-\infty}^{\infty}(f(x), \psi(x)) d x=\int_{-\infty}^{\infty} f_{m}(x) h(x) d x \neq 0$ which implies that $M$ is not minimal and the result follows.

3. The finite-dimensional case. Spectral analysis holds for $L_{1}^{H}(\boldsymbol{R})$, where $H$ is finite-dimensional. By duality, this result is a consequence of the following:

THEOREM 2. Let $H$ be finite-dimensional Hilbert space. Then every $w^{*}$-closed, invariant, nontrivial subspace of $L_{\infty}^{H}(\boldsymbol{R})$ contains an one-dimensional invariant subspace.

Proof. Let $f \in L_{\infty}^{H}(\boldsymbol{R})$ and $f_{n}(x)=\left(f(x), e_{n}\right)(n=1,2, \cdots, N)$ where $\left\{e_{n}\right\}_{n=1}^{N}$ is a basis of $H$. We may assume that $f_{1} \neq 0$ and $0 \in \operatorname{Sp}\left(f_{1}\right)$. Let $M$ denote the $w^{*}$-closed, invariant subspace of $L_{\infty}^{H}(\boldsymbol{R})$ generated by $f$. Let $\phi_{k} \in L_{1}(\boldsymbol{R})$ where $\operatorname{Supp} \hat{\phi}_{k}=[-1 / k, 1 / k] \hat{\phi}_{k}(0) \neq 0$ for $k=1,2, \cdots$, . Hence, $g_{k}(x)=\int_{-\infty}^{\infty} f(x-\alpha) \phi_{k}(\alpha) d \alpha$ is not identically zero and belongs to $M(k=1,2, \cdots$,$) . Let g_{k, n}(x)=\left(g_{k}, e_{n}\right)$ for $k=1,2, \cdots$, and $n=$ $1,2 \cdots, N$.

There exist an integer $j, 1 \leqq j \leqq N$, and a subsequence $k_{l} \rightarrow \infty$ such that

$$
\max _{1 \leq n \leq N}\left\|g_{k_{l}, n}\right\|_{L_{\infty}}=\left\|g_{k_{l}, j}\right\|_{L_{\infty}} .
$$

If $\hat{\phi}_{k_{l}}$ is multiplied by an appropriate function, it will follow that 


$$
\left\|g_{k_{l}, j}\right\|_{L_{\infty}}=1 \text { and } g_{k_{l}, j}(0)>1-\frac{1}{k_{l}} .
$$

By Bernstein's inequality [5, p. 149] we have

$$
\left\|g_{k_{l}, j}^{\prime}\right\|_{L_{\infty}} \leqq \frac{1}{k_{l}} \quad(l=1,2, \cdots,) \text {. }
$$

Hence,

$$
\left|g_{k, l}, j(x)-1\right| \leqq \frac{1}{k_{l}}(|x|+1) \quad \text { which }
$$

implies that $\left\{g_{k_{l}, j}\right\}_{l=1}^{\infty}$ converges uniformly on compact sets to the constant function 1.

By the $w^{*}$-compactness of the unit ball in $L_{\infty}(\boldsymbol{R})$ there exists a subsequence of $k_{l}$, which will be denoted again by $k_{l}$, such that

$$
g_{k_{l}, n}(x) \stackrel{w^{*}}{\longrightarrow} \psi_{n}(x) \quad n=1,2, \cdots, N
$$

where $\psi_{n} \in L_{\infty}(\boldsymbol{R})$ and $\psi_{j}(x) \equiv 1$.

Obviously, $\operatorname{Sp}\left(\psi_{n}\right) \subset\{0\}$ and by an elementary theorem on spectral synthesis (see, for instance, [1] or [4] pp. 151 and 181) we deduce

$$
\psi_{n}(x)=c_{n} \quad c_{n} \in C \quad(n=1,2, \cdots, N) .
$$

Hence, the function $\psi \in L_{\infty}^{H}(\boldsymbol{R}), \psi \neq 0$, where $\left(\psi(x), e_{n}\right)=c_{n}$ $(n=1,2, \cdots, N)$ belongs to $M$ which completes the proof of the theorem.

REMARK 1. We have verified, actually, that the analogue of Beurling's theorem [1] in spectral analysis of bounded functions on the real line, holds for $L_{\infty}^{I I}(\boldsymbol{R})$ where $H$ is finite-dimensional.

REMARK 2. Theorem 2 may be, similarly, proved for $L_{\infty}^{I I}\left(\boldsymbol{R}^{n}\right)$ where $n>1$ and $H$ is finite-dimensional.

4. The infinite-dimensional case. Spectral analysis does not hold for $L_{1}^{H}(\boldsymbol{R})$ where $H$ is infinite-dimensional. That is, there exists a proper closed, translation invariant subspace of $L_{1}^{H}(\boldsymbol{R})$ which is contained in no maximal, closed, invariant subspace of $L_{1}^{H}(\boldsymbol{R})$. We prove the following:

THEOREM 3. Let $H$ be a separable, infinite-dimentional Hilbert space. There exists a nontrivial, $w^{*}$-closed, invariant subspace of $L_{\infty}^{H}(\boldsymbol{R})$ which does not contain any one-dimensional, invariant subspace. 
For the proof of Theorem 3 we will need the following lemma:

LeMma 4. Let $f_{1}$ and $f_{2}$ be in $L_{\infty}(\boldsymbol{R}) \cap L_{1}(\boldsymbol{R})$ such that $\hat{f}_{1}$ is a constant $d$ in the interval $[a, b]$.

If $\phi_{\tau}, \tau \in \Gamma$, is a net in $L_{1}(\boldsymbol{R})$ such that

$$
\left(f_{i} * \dot{\phi}_{\tau}\right)(x) \stackrel{w^{*}}{\tau} a_{i} e^{i \lambda x} \quad(i=1,2)
$$

where $a<\lambda<b$, then we have

$$
a_{1} \hat{f}_{2}(\lambda)=a_{2} d \text {. }
$$

Proof. We may assume that $\operatorname{Supp} \hat{\phi}_{\tau} \leqq[a, b]$ for every $\tau \in \Gamma$. Hence $f_{1} * \phi_{\tau}=d \phi_{\tau}$ for any $\tau \in \Gamma$. Suppose that $d \neq 0$. Then $\phi_{\tau} \stackrel{w^{*}}{\rightarrow}$ $\left(a_{1} / d\right) e^{i \lambda x}$ and

$$
f_{2} * \phi_{\tau} \stackrel{w^{*}}{\longrightarrow} \frac{a_{1}}{d} \widehat{f}_{2}(\lambda) e^{i \lambda x}
$$

If $d=0$, then $f * \phi_{\tau}=0$ for any $\tau \in \Gamma$ and we have $a_{1}=0$. This completes the proof of the lemma.

For $h \geqq 0, q>p$ let $T_{h, p, q}(x)$ be the function:

$$
T_{h, p, q}(x)=\left\{\begin{array}{lc}
\frac{3 h}{q-p}(x-p) & p \leqq x<\frac{2}{3} p+\frac{1}{3} q \\
h & \frac{2}{3} p+\frac{1}{3} q \leqq x<\frac{1}{3} p+\frac{2}{3} q \\
\frac{3 h}{p-q}(x-q) & \frac{1}{3} p+\frac{2}{3} q \leqq x<q \\
0 & \text { elsewhere . }
\end{array}\right.
$$

The proof of Theorem 3. Let $\chi_{n}(x)=T_{h_{n}, p_{n}, q_{n}}(x)$ satisfy the following conditions:

$$
\begin{gathered}
h_{1}=1, \quad p_{1}=-1 \quad \text { and } \quad q_{1}=2 . \\
q_{n}-p_{n}=\frac{3}{n \lg n} \quad \text { and } \quad h_{n}=\lg n \quad(n=2,3, \cdots,) .
\end{gathered}
$$

(iii) For each $\lambda, 0<\lambda<1$, there exists a sequence $n_{k} \rightarrow \infty$, such that $\lim _{k \rightarrow \infty} \chi_{n_{k}}(\lambda)=\infty$.

Let $g_{n}^{*}$ be the sequence defined by

$$
\hat{g}_{n}^{*}(x)=\chi_{n}(x) \quad(n=1,2, \cdots,) .
$$


Let $g_{n}=g_{n}^{*} * \psi$ where $\psi \in L_{1}(\boldsymbol{R}),\|\psi\|_{L_{1}}=1$ and Supp $\hat{\psi} \subset[0,1]$. By condition (ii) we have $\left\|g_{n}\right\|_{L_{\infty}} \leqq 2 / n(n=2,3, \cdots)$. Hence there exists a function $f \in L_{\infty}^{H}(\boldsymbol{R})$ such that $\left(f(x), e_{n}\right)=g_{n}(x)$ for $n=$ $1,2, \cdots$, where $\left\{e_{n}\right\}_{n=1}^{\infty}$ is a basis of $H$.

Suppose that the $w^{*}$-closed, invariant subspace generated by $f$ contains an one-dimensional invariant subspace. That is, there exist a net $\phi_{\tau}, \tau \in \Gamma, \phi_{r} \in L_{1}(\boldsymbol{R})$ and a real number $\mu$ such that

$$
\left(g_{n} * \phi_{\tau}\right)(x) \underset{\tau}{\stackrel{w^{*}}{\longrightarrow}} a_{n} e^{i \mu x} \quad(n=1,2, \cdots,)
$$

where $\left\{a_{n}\right\}_{n=1}^{\infty} \in l_{2}$. For every $g_{n}$ we have $\operatorname{Sp}\left(g_{n}\right) \subset[0,1]$. Hence, we may assume that $\mu \in(0,1)$.

From (1) we have $g_{n}^{*} *\left(\psi * \phi_{\tau}\right) \underset{\tau}{\stackrel{w^{*}}{\rightarrow}} a_{n} e^{i \mu x}(n=1,2, \cdots$,$) .$

By (iii) there exists a sequence $n_{k} \rightarrow \infty$ such that $\lim _{k \rightarrow \infty} \chi_{n_{k}}(\mu)=\infty$. By Lemma 4 we deduce that $a_{n}=a_{1} \chi_{n}(\mu)(n=1,2, \cdots$,$) which$ implies that $a_{n}=0$ for each $n$. This completes the proof of the theorem.

5. Spectral analysis in subgroups of the motion group. In [5] it was verified that the one-sided analogue of Wiener's theorem fails to hold for the motion group. However, we will prove that the one-sided Wiener's theorem holds for the subgroup $M_{K}$ where

$$
M_{K}=\left\{\left(\begin{array}{ll}
e^{i k \theta} & z \\
0 & 1
\end{array}\right): \theta=\frac{2 \pi}{K}, k=0,1,2, \cdots, K-1, z \in C\right\} .
$$

(See also [3].)

By duality, this result is a consequence of the following:

THEOREM 4. Every $w^{*}$-closed, right invariant, nontrivial subspace of $L_{\infty}\left(M_{K}\right)$ contains an irreducible (minimal) right invariant, nontrivial subspace.

Proof. Let $f \in V, f \neq 0$, where $V$ is a $w^{*}$-closed, right invariant subspace of $L_{\infty}\left(M_{K}\right)$. The subspace $V$ contains all functions $g$ such that $g\left(e^{i k \theta}, z\right)=f\left(e^{i(k+m) \theta}, z-w e^{i k \theta}\right)$ where $m \in Z$ and $w \in C$. For a suitable $r \in \boldsymbol{Z}$ the function

$$
\sum_{m=0}^{K-1} f\left(e^{i(k+m) \theta}, z\right) e^{-i r m \theta}=e^{i r k \theta} \sum_{m=0}^{K-1} f\left(e^{i m \theta}, z\right) e^{-i m \theta}=e^{i r k \theta} P(z)
$$

is nonzero and belongs to $V$. Let $P_{s}(z)=P\left(e^{i s} z\right)$ for $s=0,1, \cdots$, $K-1$. Then by Theorem 2 and Remark $2\left(P_{8}\right.$ are looked upon as the coordinates of a function in $L_{\infty}^{H}\left(\boldsymbol{R}^{2}\right)$ where $H$ is $K$-dimensional), there exist $\psi_{n} \in L_{1}\left(\boldsymbol{R}^{2}\right)(n=1,2, \cdots, \lambda), \lambda \in C$ and $a_{s} \in C(s=0,1, \cdots, K-1)$ where $\sum_{s=0}^{K-1}\left|a_{s}\right|>0$, such that 


$$
\int_{R^{2}} P_{s}(z-\xi) \psi_{n}(\xi) \stackrel{w^{*}}{\longrightarrow} a_{s} e^{i(\lambda, z)} .
$$

(Here, for $z_{1} z_{2} \in \boldsymbol{C},\left(z_{1}, z_{2}\right)=x_{1} x_{2}+y_{1} y_{2}$ where $z_{1}=x_{1}+i y_{1}, z_{2}=x_{2}+i y_{2}$.) Let $\chi_{n}(\xi)=\sum_{s=0}^{K-1} \psi_{n}\left(e^{-i s \theta} \xi\right) \quad n=1,2, \cdots$, Obviously, $\chi_{n}(\xi)=\chi_{n}\left(e^{i s \theta} \xi\right)$ for $s=0,1, \cdots, K-1$. Then, by (3), we have

$$
\int_{R^{2}} P(z-\xi) \chi_{n}(\xi) d \xi \stackrel{w^{*}}{\longrightarrow} \sum_{s=0}^{K-1} a_{s} e^{i\left(e^{-i s} \lambda_{, z)}\right.} \text {. }
$$

Hence, by (2), the function

$$
e^{i r k \theta} \int_{R^{2}} P\left(z-\xi e^{i k \theta}\right) \chi_{n}(\xi) d \xi=e^{i r k \theta} \int_{R^{2}} P(z-\xi) \chi_{n}(\xi) d \xi
$$

belongs to $V$ for each $n$. Finally, by (4), the function $Q \in L_{\infty}\left(M_{K}\right)$ where $Q\left(e^{i k \theta}, z\right)=e^{i r k \theta} \sum_{s=0}^{K-1} a_{s} e^{i\left(e^{-i s} \theta_{\lambda, z)}\right.}$ belongs to $V$. Arguing as in [5], it can be verified that the $w^{*}$-closed, right invariant subspace generated by $Q$ irreducible. This completes the proof.

\section{REFERENCES}

1. A. Beurling, Un théorème sur les fonctions bornees et uniformement continues sur l'axe reel, Acta Mathematica, 77 (1945), 127-136.

2. Y. Domar, Spectral analysis in spaces of sequences summable with weights, J. Functional Analysis, 5 (1970), 1-13.

3. H. Leptin, On one-sided harmonic analysis in non-commutative locally compact groups,

J. Reine und Angew. Math., 306 (1979), 122-153.

4. L. Loomis, Abstract Harmonic Analysis, Van Nostrand, New York, 1953.

5. Y. Meyer, Algebraic Numbers and Harmonic Analysis, North Holland, Amsterdam, 1972.

6. Y. Weit, On the one-sided Wiener's theorem for the motion group, Ann. of Math., 111 (1980), 415-422.

Received July 11, 1979.

UnIVERSITY OF HAIFA

HaIfa, ISRAEL 


\section{PACIFIC JOURNAL OF MATHEMATICS}

\section{EDITORS}

DoNALD BABBITT (Managing Editor)

University of Galifornia

Los Angeles, California 90024

HUgo RossI

University of Utah

Salt Lake City, UT 84112

C. C. MOORE AND ANDREW OGG

University of California

Berkeley, CA 94720
J. DugunduI

Department of Mathematics

University of Southern California

Los Angeles, California 90007

R. Finn and J. Milgram

Stanford University

Stanford, California 94305

\section{ASSOCIATE EDITORS}
E. F. BeCKenbaCh
B. H. NeumanN
F. WOLF
K. YoSHIDA

\section{SUPPORTING INSTITUTIONS}

UNIVERSITY OF BRITISH COLUMBIA

CALIFORNIA INSTITUTE OF TECHNOLOGY

UNIVERSITY OF CALIFORNIA

MONTANA STATE UNIVERSITY

UNIVERSITY OF NEVADA, RENO

NEW MEXICO STATE UNIVERSITY

OREGON STATE UNIVERSITY

UNIVERSITY OF OREGON
UNIVERSITY OF SOUTHERN CALIFONIA STANFORD UNIVERSITY UNIVERSITY OF HAWAII UNIVERSITY OF TOKYO UNIVERSITY OF UTAH WASHINGTON STATE UNIVERSITY UNIVERSITY OF WASHINGTON 


\section{Pacific Journal of Mathematics}

\section{Vol. 91, No. $1 \quad$ November, 1980}

Harvey Leslie Abbott, Extremal problems on nonaveraging and nondividing

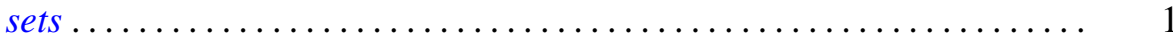

Marine Bruce Abrahamse and Stephen D. Fisher, Mapping intervals to

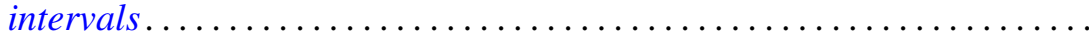

William Wells Adams, The best two-dimensional Diophantine

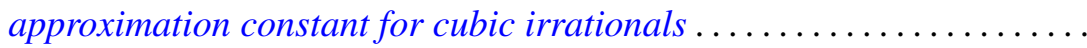

Marilyn Breen, A quantitative version of Krasnosel'skiu 's theorem in

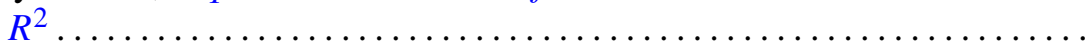

Stephen LaVern Campbell, Linear operators for which $T^{*} T$ and $T T^{*}$

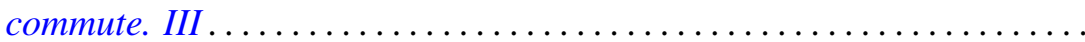

Zvonko Cerin, On cellular decompositions of Hilbert cube manifolds ......

J. R. Choike, Ignacy I. Kotlarski and V. M. Smith, On a characterization

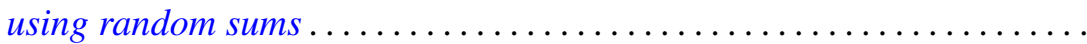

Karl-Theodor Eisele, Direct factorizations of measures .............. 79

Douglas Harris, Every space is a path component space ............. 95

John P. Holmes and Arthur Argyle Sagle, Analytic H-spaces, Campbell-Hausdorff formula, and alternative algebras.............

Richard Howard Hudson and Kenneth S. Williams, Some new residuacity criteria ..........................................

V. Karunakaran and Michael Robert Ziegler, The radius of starlikeness for a class of regular functions defined by an integral ....

Ka-Sing Lau, On the Banach spaces of functions with bounded upper

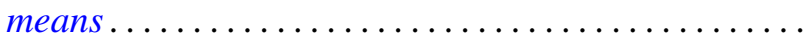

Daniel Paul Maki, On determining regular behavior from the recurrence formula for orthogonal polynomials................

Stephen Joseph McAdam, Asymptotic prime divisors and going down...

Douglas Edward Miller, Borel selectors for separated quotients ..

Kent Morrison, The scheme of finite-dimensional representations of an algebra

Donald P. Story, A characterization of the local Radon-Nikodým property by tensor products

Arne Stray, Two applications of the Schur-Nevanlinna algorithm ...

N. B. Tinberg, The Levi decomposition of a split $(B, N)$-pair ...

Charles Irvin Vinsonhaler and William Jennings Wickless, A theorem on quasi-pure-projective torsion free abelian groups of finite rank... 